\title{
Ärztemangel: Ist die Lösung weiblich?
}

Der Ärztemangel in der Schweiz wird immer deutlicher. Hat man am Anfang dieses Jahrhunderts noch von einer Ärzteschwemme gesprochen, zeigt der Trend heute eindeutig in die umgekehrte Richtung. Trotz der Anerkennung von 210 ausländischen Diplomen [1] blieben im Jahr 2010 zahlreiche Stellen unbesetzt. Eine wichtige Rolle spielen dabei das Geschlechterverhältnis bei den Studierenden und die steigende Quote der diplomierten Ärztinnen.

Esther Bieri

Esther Bieri aus Luzern ist Supervisorin und Coach FH IAP. Sie ist als Projektleiterin in Schweizer Spitälern tätig.

* Name von der Redaktion geändert.

Korrespondenz:

FMH Kommunikation Jacqueline Wettstein

Elfenstrasse 18

CH-3000 Bern 15

Tel. 0313591111

Fax 0313591112

kommunikation(at)fmh.ch
Über Jahre richteten sich die Arbeitszeiten nach den Bedürfnissen der Klinik und waren entsprechend nach oben unbegrenzt. 2005 wurde in den Spitälern die Arbeitszeit für Assistenz- und teilweise auch für Oberärzte auf 50 Stunden pro Woche begrenzt.

Andererseits nimmt der Bedarf an medizinischen Leistungen mit steigender Lebenserwartung der Bevölkerung zu. Entsprechend mussten zusätzliche Stellen geschaffen werden. Zunehmend zeigt sich, dass zahlreiche Assistenz- und Kaderarztstellen nicht mehr besetzt werden können. In einigen Fachrichtungen, zum Beispiel der Hausarztmedizin - mangelt es also bereits heute an Nachwuchs.

Dabei gilt das Medizinstudium nach wie vor als attraktiv. Entsprechend ist die Nachfrage nach den Studienplätzen ungebrochen gross: Für das Studienjahr 2011/2012 haben sich an den Universitäten Basel, Bern, Freiburg und Zürich 2936 Personen für das Studium der Humanmedizin angemeldet. Das sind dreieinhalb Mal mehr Anmeldungen als Studienplätze. Die Verbindung der Schweizer Ärztinnen und

\section{FMH: konkrete Projekte gefordert}

Die Verbindung der Schweizer Ärztinnen und Ärzte FMH sieht eine Ursache des Ärztemangels darin, dass zu wenig Ärzte ausgebildet werden. Ein Kapitel ihres Zielpapiers für die Legislaturperiode 2008 bis 2012 [3] widmet sie denn auch der «Förderung des beruflichen Nachwuchses». Sie setzt sich auf den verschiedenen politischen Ebenen für eine Erhöhung der Studierendenzahl von 20\% ein.

Die zweite wichtige Ursache des Ärztemangels liegt im Bedürfnis der jungen Ärztinnen und Ärzte, Familie und medizinische Laufbahn zu vereinbaren. Viele unterbrechen ihre Karriere oder steigen zum Zeitpunkt der Familiengründung sogar ganz aus dem Beruf aus. Die FMH unterstützt deshalb konkrete Projekte ihrer Mitglieder, die zeitgemässe und familienfreundliche Arbeitsbedingungen in den Institutionen des Gesundheitswesens (Horte, Teilzeitarbeit usw.) zum Ziel haben.
Ärzte FMH fordert deshalb, dass die Anzahl Studienplätze um 20 Prozent angehoben werden muss.

\section{Medizinstudium: $63 \%$ Frauen}

Ein Blick in die Studierendenstatistik der Universität Zürich macht eine Feminisierung im Fach Humanmedizin deutlich. Bereits im Jahr 2000 lag der Frauenanteil bei $63 \%$. Nur: Haben Ärztinnen dieselben Karrierechancen wie ihre männlichen Kollegen? Wie gut ist eine medizinische Laufbahn mit der Gründung einer Familie vereinbar? Eine Untersuchung des UniversitätsSpitals Zürich zur gleichberechtigten Nachwuchsförderung von Ärztinnen und Ärzten [2] zeichnet ein klares Bild: Der Frauenanteil bei den Assistenzärzten liegt bei $40 \%$, auf der Stufe Oberärztinnen noch bei 30\%. Weiter oben auf der Karriereleiter sind Frauen eine Seltenheit: $8 \%$ bei Leitenden Ärzten und Ärztinnen.

\section{Ehemann übernimmt Rolle des Familien- und Hausmanns}

Dr. med. Karin Alpiger* ist heute Mutter von zwei schulpflichtigen Kindern. 1993 schloss sie ihr Medizinstudium ab. Für sie war immer klar, dass sie nach ihrem Praxisjahr eine chirurgische Laufbahn einschlagen würde. Während ihrer Zeit als Assistenzärztin - in der sie die Ausbildungsjahre und die nötige Anzahl Operationen für den Facharzttitel FMH Chirurgie absolvierte - wurde die talentierte Ärztin von ihren Chefs gefördert. «Mit dem Fortschritt der Karriere liess diese Förderung jedoch nach», erinnert sich Alpiger. Stattdessen hat man ihr vermehrt unliebsame Aufgaben übertragen, die sie im Erlernen des Basishandwerks behinderten. «Während meiner Funktion als Oberärztin kamen meine beiden Kinder zur Welt. Die komplikationslosen Schwangerschaften liessen es zu, dass ich bis zum Geburtstermin im Vollzeitpensum arbeiten konnte. Glücklicherweise hatte mein Ehemann die Grösse, die Rolle des Familien- und Hausmanns zu übernehmen. Er hat als IT-Manager eines grossen Verlagshauses seine Karriere schon gemacht und wollte mir nun meine Laufbahn im Spital ermöglichen.» 


\section{Teilzeitpensum für Kaderärzte: sinnvoll?}

Für Karin Alpiger kam ein Teilzeitpensum nie in Frage. «Chirurgie ist ein Handwerk, das viel Übung und Erfahrung verlangt. Mit einem reduzierten Pensum hätte ich für die Oberarztreife entsprechend länger gebraucht», meint sie. Heute, da sie eine eigene Praxis betreibt, kann sie die Arbeitszeiten besser nach ihrer Familie ausrichten. Trotzdem arbeitet sie Vollzeit. «Nach einer so langen Ausbildung habe ich mir eine Menge Knowhow und Erfahrung angeeignet. Ich habe mich entschieden, beides zugunsten meiner Patientinnen und Patienten zu verwenden.» Zwar versteht Karin Alpiger den Wunsch junger Ärztinnen und Ärzten sich die Kombination von Beruf und Familie im Rahmen einer Teilzeitstelle zu ermöglichen. «Gerade vor dem Hintergrund des Ärztemangels sollte man den drohenden Knowhow-Verlust bei der Entscheidung über die Pensumgrösse berücksichtigen.»

\section{Kinderbetreuung bis 22.00 Uhr}

Bis heute gibt es nur wenige Kliniken, die in einem chirurgischen Fach Teilzeitstellen für Kaderärztinnen und -ärzte geschaffen haben. Eine davon ist die Orthopädie des Luzerner Kantonsspitals in Wolhusen. Hier teilen sich zwei Frauen und ein Mann zwei Oberarztstellen. Alle drei haben Familie. «Ich habe das Talent dieser angehenden Fachärzte gesehen und wollte sie weiterhin fördern - unabhängig vom Geschlecht», sagt Chefarzt Dr. med. Richard F. Herzog. «Ja, es ist ein Experiment. Es funktioniert nur dann, wenn die Oberärzte mit Teilzeitpensen bereit sind, die Arbeitszeiten genauso einzuhalten wie alle anderen Kollegen auch.» Der Personalbestand einerseits und die Tagesstruktur der Klinik andererseits lassen es nicht zu, das Haus bereits um 18.00 Uhr zu verlassen. Als Kaderarzt trägt man für seine Patienten die gesamte Verantwortung. Es wird gearbeitet, bis der letzte Patient untersucht und der letzte OP-
Bericht diktiert ist. «Deshalb sind die Spitäler gefordert, endlich Kinderbetreuung von 6.00 bis $22.00 \mathrm{Uhr}$ $\mathrm{zu}$ organisieren. Diese muss vom Arbeitsplatz des Elternteils gut erreichbar sein.» Im Gegensatz zu Karin Alpiger glaubt Chefarzt Herzog, dass Teilzeitarbeit eine wichtige Rolle spielen wird, wenn es darum geht, dem Ärztemangel nachhaltig zu begegnen. Nur so könnten Mütter mit einem chirurgischen Facharzt-Titel dem Gesundheitswesen als Leistungserbringerinnen langfristig erhalten bleiben. «Dazu braucht es allerdings angepasste Arbeitsbedingungen, wie die Möglichkeit der familienexternen Kinderbetreuung oder der Spezialisierung in einem Teilbereich.»

\section{Koordination der Ausbildung bis zur Facharztreife}

Sowohl Karin Alpiger wie auch Richard F. Herzog wünschen sich von der FMH und von den Fachgesellschaften, dass sie die Schaffung von Ausbildungsregionen unterstützen. Diese müssten aus verschieden grossen Spitälern bestehen, damit die Kandidatinnen und Kandidaten ihre Ausbildungsjahre bis zur Facharztreife planen können. Gerade die Ausbildung von Kandidatinnen in Teilzeitpensen ist für die Klinikverantwortlichen besonders anspruchsvoll. Eine langfristige Planung ist für das Spital eine wichtige Voraussetzung, um Personalengpässe zu vermeiden und dem zunehmenden Facharztmangel zu begegnen.

\section{Literatur}

1 Suter P, de Haller J, Täuber M, Gassmann B. Zukünftiger Bedarf an Ärztinnen und Ärzten in der Schweiz. Schweiz Ärztezeitung. 2006;87(17):713-4.

2 Jacquemart C, Boos L. Gleichberechtigte Nachwuchsförderung von Ärztinnen und Ärzten am Universitätsspital Zürich; 2000

3 Strategische Ziele der FMH für die Legislaturperiode 2008-2012, www.fmh.ch

\section{Drei Ärztinnen, drei Erfahrungsberichte}

\author{
Prof. Dr. med. Barbara Buddeberg-Fischer, \\ Professorin und Fachärztin Psychiatrie \\ und Psychotherapie FMH und Kinder- und \\ Jugendpsychiatrie und -psychotherapie FMH \\ sowie Fähigkeitsausweis für Psychosomatische \\ und Psychosoziale Medizin (SAPPM) \\ Ich befinde mich auf der letzten Etappe meiner beruf- \\ lichen Tätigkeit. Wenn ich zurückblicke, dann staune \\ ich, wie ich alles unter einen Hut gebracht habe: den \\ Erwerb von zwei Facharzttiteln, meine Praxistätigkeit \\ und parallel dazu die Kindererziehung und meine \\ wissenschaftliche Tätigkeit. Bereits früh habe ich mir
}

ein Standbein in der sozialwissenschaftlichen Forschung aufgebaut und zum Beispiel Studien mit Jugendlichen zu Essstörungen durchgeführt. Als Forscherin finde ich es schade, dass die Wissenschaft noch immer männerorientiert ist und Frauen seltener diesen Einstieg suchen. Während sich 12 Prozent der Männer für eine medizinisch-akademische Berufslaufbahn entscheiden, schlagen nur gerade 2 Prozent der Medizinerinnen diese Richtung ein. Und auch auf den Chefetagen der Spitäler sind die Frauen immer noch stark untervertreten. Ich hoffe, dass sich dies in den nächsten Jahren ändern wird, schliessen 
doch seit einigen Jahren mehr Frauen als Männer das Studium der Humanmedizin ab.

Ich finde es eine positive Entwicklung, dass vor allem jüngere Ärztinnen und Ärzte nicht mehr bereit sind, rund um die Uhr zu arbeiten. Doch leider sind die Weiterbildung und die Anstellungsbedingungen vielfach noch an männlichen Lebensläufen orientiert. Die 50-Stunden-Woche in der Weiterbildungsphase wird in vielen Spitälern und manchen Fachbereichen nicht eingehalten. Ebenso richten sich die Kinderkrippen von Spitälern oft nicht nach den Bedürfnissen von Ärztinnen, zum Beispiel in Bezug auf ihre Arbeitszeiten. Dies muss unbedingt geändert werden. Gerade für Frauen braucht es viel mehr Teilzeitstellen von etwa 80 bis 60 Prozent, damit sich Beruf und Familie kombinieren lassen. Und auch die Weiterbildung ist meiner Ansicht nach in einem 80-Prozent-Pensum machbar, selbst in der Chirurgie. $\mathrm{Ob}$ jemand eine gute Ärztin oder ein guter Arzt ist, entscheidet sich bekanntlich nicht an der Anzahl Überstunden, sondern u.a. an der Persönlichkeit. Medizinstudentinnen und ihren Kollegen rate ich, sich möglichst früh zu entscheiden, welchen Facharzt sie erwerben wollen. Damit lässt sich die Weiterbildung optimal und effizient gestalten. Denkbar wäre zum Beispiel, dass die Weiterbildung zentral organisiert ist: Ein Jahr vor dem Staatsexamen entscheiden sich die Medizinstudierenden für eine Fachrichtung und melden sich bei der entsprechenden zentralen Stelle. Von dieser erhalten sie dann verschiedene Angebote für die gesamte Weiterbildungs-

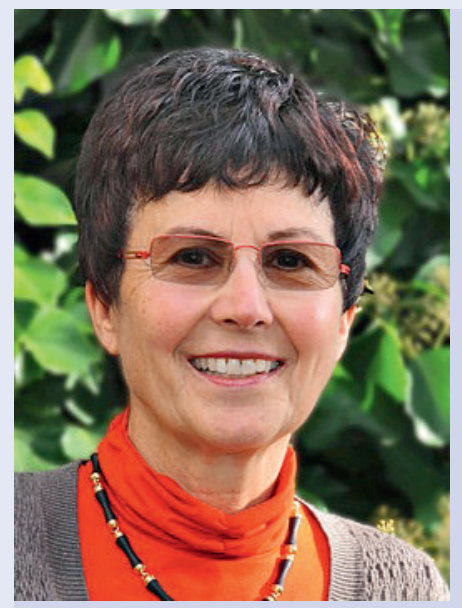

Barbara Buddeberg-Fischer: «Heute schliessen mehr Frauen als Männer das Medizinstudium ab. Darum sind Veränderungen notwendig in der Weiterbildung, in den institutionellen Rahmenbedingungen in den Spitälern, in der Berufswelt und auch in der Forschung.»

zeit, auf die sie sich bewerben können. Meiner Meinung nach dauert die ärztliche Weiterbildung derzeit zu lange. Man könnte sie ohne Qualitätseinbusse auf vier bis fünf Jahre beschränken und jeweils zu erreichende Jahresziele definieren. Die Weiterbildung setzt sich ja in die kontinuierliche Fortbildung während der ganzen Berufstätigkeit fort. Eine verkürzte, effizienter gestaltete Weiterbildung käme vor allem auch den Frauen in unserem Beruf zugute.
Dr. med. Brigitte Muff, Chefärztin Chirurgie, Fachärztin für Chirurgie FMH mit Schwerpunkten Viszeralchirurgie sowie Allgemein- und Unfallchirurgie und Fähigkeitsausweis psychosoziale und psychosomatische Medizin

Ich bin seit über 20 Jahren Chirurgin und seit 2005 als Chefärztin an der Chirurgischen Klinik im Spital Bülach tätig. Mein Beruf bereitet mir auch nach all den Jahren immer noch grosse Freude, die operative Tätigkeit sowie der Kontakt zu den Patienten sind dafür die Hauptgründe. Weiter liegt mir das Einführen junger Ärztinnen und Ärzte in den Klinikalltag und das Lehren des chirurgischen Handwerks sehr am Herzen. Natürlich gibt es auch in unserem Beruf Schattenseiten. Diese sind aber nicht unbedingt wie oftmals vermutet die hohen Präsenzzeiten oder die anstrengenden Dienste, sondern die Tatsache, dass immer mehr Personen, die mit unserem Kerngeschäft nichts zu tun haben, uns diktieren (wollen), was wir tun und lassen sollen. Der Verwaltungsapparat in den Spitälern hat sich in den letzten Jahren massiv vergrössert. Dadurch resultieren zwangsläufig mehr (sinnlose) Schnittstellen mit der Ärzteschaft. Die ständigen Rechtfertigungen für unser Tun gegenüber allen möglichen Bereichen im Spital sind je-

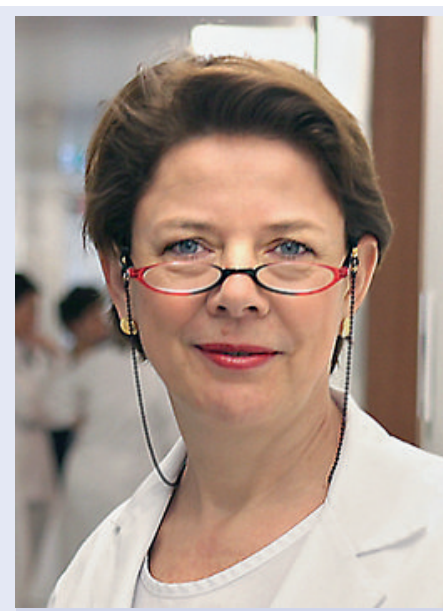

Brigitte Muff: «Mein Beruf bereitet mir auch nach all den Jahren immer noch grosse Freude, die operative Tätigkeit sowie der Kontakt zu den Patienten sind dafür die Hauptgründe.»

doch zermürbend, demotivierend und sicher nicht qualitätsfördernd.

Spitäler werden durch Manager geführt, was zwangsläufig einen Paradigmenwechsel zur Folge hat. Mit ihrem betriebswirtschaftlichen Hintergrund sind sie 
überzeugt, dass Spitäler als Dienstleistungsbetriebe gleich geführt werden können und sollen wie irgendwelche Sachgüterproduktionsbetriebe*. Früher gab es kranke Menschen, also Patienten, die eine Therapie benötigten. Die daraus entstandenen Kosten wurden über Subventionen, Prämien oder direkt beglichen. Heute spricht man von Kunden, und im Zentrum steht nicht mehr die sinnvollste Therapie für den Einzelnen, sondern die Gewinnoptimierung für den Betrieb. Ein sozialer Auftrag soll nach marktwirtschaftlichen Regeln umgesetzt werden. Eine paradoxe Situation, in der wir uns befinden.

Als Frau und Mutter von zwei fast erwachsenen Töchtern werde ich oft mit der Frage konfrontiert, ob und wie ich Familie und Arztberuf unter einen Hut bringe. Diese Frage sollte heute eigentlich gar nicht mehr relevant sein. Vielmehr sollte es eine Selbstverständlichkeit sein, dass Mütter auch leitende Funktionen in sämtlichen Berufsrichtungen innehaben. Natürlich müssen dafür gewisse Voraussetzungen erfüllt sein: Zunächst braucht es die Erkenntnis seitens der Eltern, aber auch der Bevölkerung, dass eine Fremdbetreuung der Kinder nichts Schlechtes ist. Es müssen genügend Krippenplätze vorhanden sein, und die Betreuung sollte auch für Familien gewährleistet sein, in denen einer der Eltern oder beide im Schichtbetrieb arbeiten. Dieses Modell wird übrigens in Frankreich oder in den skandinavischen Ländern bereits angewendet. Heute sind Ärztinnen in leitender Spitalfunktion oder in der Lehre klar untervertreten. Wenn frau jedoch will (und das ist die wichtigste Voraussetzung) und die Spitalwelt nicht von vornherein als frauen- und mütterfeindlich akzeptiert, dann kann dieses geschlechtliche Ungleichgewicht korrigiert werden. Man kann dafür auch Jobsharing-Modelle anwenden, wie es beispielsweise die Maternité des Stadtspitals Triemli in Zürich erfolgreich tut durch zwei Chefärztinnen mit Teilpensen.

Für die kommende Ärztegeneration hoffe ich, dass medizinfremde Diktate wie beispielsweise das Arbeitsgesetz für Ärzte in Weiterbildung (für operative Disziplinen eher hinderlich) oder das Ausfüllen von Formularen für irgendwelche Pseudoqualitätssicherung nicht weiter zunehmen. Ferner müsste die Ökonomisierung in der Medizin gestoppt werden, sodass der Patient wieder im Mittelpunkt steht und nicht der dienstleistungskaufende Kunde. Ich für meine Person kann mir gut vorstellen, irgendwann in Zukunft auch in einem Krisengebiet oder Entwicklungsland tätig zu sein. Genau dort eben, wo es darum geht, kranken Menschen zu helfen ohne ökonomische Hintergedanken.

* Dieses Phänomen beschreibt Mathias Binswanger in seinem Buch «Sinnlose Wettbewerbe: Warum wir immer mehr Unsinn produzieren.», Herder-Verlag, sehr schön. Ebenso widmet sich Bernard Lown in «Die verlorene Kunst des Heilens. Anstiftung zum Umdenken», Suhrkamp-Verlag, diesem Thema.

\section{Patrizia Kündig, Medizinstudentin an der medizinischen Fakultät Basel}

Ich studiere im 5. Studienjahr Humanmedizin an der Universität Basel. Für mich war bereits vor der Matura klar, dass ich diese Richtung einschlagen werde. Und ich bin nach wie vor sehr zufrieden mit meiner Wahl. Generell würde ich mir wünschen, dass die praktische Tätigkeit auch bereits während der Ausbildung ein stärkeres Gewicht hätte. Zwar sieht das Studium mehr Praxis vor als noch vor einigen Jahren, aber diese findet leider meist nur im Rahmen einzelner Stunden statt. Die Form der Vorlesung hat immer noch einen sehr hohen Stellenwert. Auch wenn diese Art der Vermittlung ihre Berechtigung hat für manchen Lernstoff, so ist sie wahrscheinlich nicht in jedem Fall die beste Methode. Man täte gut daran, diese didaktische Form zu überdenken und das bestehende Curriculum dahingehend zu analysieren, welche Inhalte sinnvollerweise als Vorlesung präsentiert und welche Themen wie viele Stunden gelehrt werden. Eine problemorientierte oder eine praktische Vermittlung der Lerninhalte wäre in manchen Fächern angezeigt. Wichtig ist für mich und meine Kolleginnen und Kollegen vor allem, dass die Lernziele so gewichtet sind, dass sie unserer späteren

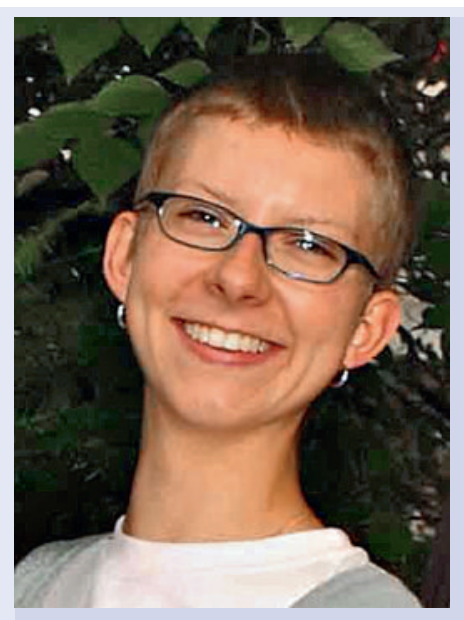

Patrizia Kündig: «Es muss ein Umdenken stattfinden, und die Arbeitsbedingungen für Ärzte und Ärztinnen müssen den heutigen Bedürfnissen angepasst werden.»

ärztlichen Tätigkeit im heutigen Gesundheitswesen entsprechen. Ich habe mich noch nicht entschieden, in welchem Fachbereich ich später tätig sein möchte. Am meisten interessiert mich gegenwärtig die Notfallmedizin, deshalb sehe ich mich z.B. in der Inten- 
sivmedizin oder bei der Rega. Und ich plane, meinen Facharzt möglichst rasch zu erwerben. Weil ich die medizinische Ausbildung mitgestalten will, engagiere ich mich bereits heute auch verbandspolitisch im Vorstand der Swiss Medical Students' Association (swimsa).

Auch nach meinem Studium möchte ich weiterhin in der Standespolitik und in der ärztlichen Aus- und Weiterbildung aktiv sein. Ich kann mir auch gut vorstellen, eine Familie zu haben, und hoffe, dass sich Kinder und die ärztliche Tätigkeit kombinieren lassen, da ich meinen Beruf nicht aufgeben möchte. Weil auch viele andere Medizinstudentinnen und -studenten später weder auf Beruf noch auf Familie verzichten wollen, muss endlich auch im Arztberuf Teilzeitarbeit möglich sein und gefördert werden. Viele Ärztinnen und auch immer mehr Ärzte wollen heute Teilzeit arbeiten, ohne dass sie als arbeitsscheu angesehen werden und auf das Karriereabstellgleis geraten. Zudem braucht es mehr Kinderkrippen an
Spitälern, und deren Angebote müssen ausgebaut und den Bedürfnissen der Ärztinnen und Ärzte angepasst werden. Ebenso möchte die jüngere Generation von Ärztinnen und Ärzten nicht ständig auf Freizeit und damit Erholung verzichten müssen. Es tut somit dringend Not, dass die gesetzlich geregelte maximale Arbeitszeit für Assistenzärztinnen und -ärzte von 50 Stunden nicht nur auf dem Papier steht, sondern auch eingehalten wird. Weil heute schon mehr Frauen ein Medizinstudium absolvieren als Männer, weil die Bedürfnisse von angehenden Ärztinnen und Ärzten sich geändert haben, braucht es einen Wandel in der ärztlichen Berufswelt und vor allem mehr Flexibilität vonseiten der Arbeitgeber. Trotzdem, auch wenn die Strukturen und die Arbeitsbedingungen in anderen Berufen komfortabler sind, bin ich nach wie vor begeistert von der Medizin, freue mich auf meine spätere Tätigkeit als Ärztin und wünsche mir, dass mir genügend Zeit bleiben wird für den individuellen Patienten.

Swiss Quality Award

INNOVATIONS IN HEALTHCARE

JETZT PROJEKTE EINREICHEN! BIS 29.02.2012

Der Swiss Quality Award prämiert herausragende Qualitätsinnovationen im Gesundheitswesen. Machen Sie mit, melden Sie jetzt Ihr Projekt auf www.swissqualityaward.ch an. 